\title{
Evasão em Intervenções Psicológicas com Pais de Crianças e Adolescentes: Relato de Experiência
}

\author{
Angela Helena Marin ${ }^{1}$ \\ ${ }^{1}$ Universidade Federal do Rio Grande do Sul, RS, Brasil. \\ Magda Pozzobon ${ }^{3}$ \\ ${ }^{3}$ Universidade do Vale do Rio dos Sinos, RS, Brasil.
}

\author{
Patrícia Alvarenga ${ }^{2}$ \\ ${ }^{2}$ Universidade Federal da Bahia, BA, Brasil. \\ Taiane Costa de Souza Lins ${ }^{4}$ \\ ${ }^{4}$ Universidade Federal da Bahia, BA, Brasil.
}

João Marcos de Oliveira ${ }^{5}$

${ }^{5}$ Universidade Federal da Bahia, BA, Brasil.

\begin{abstract}
Resumo: A evasão de participantes em pesquisas que avaliam efeitos de intervenções com pais de crianças e adolescentes tem sido pouco investigada pelos pesquisadores brasileiros, que enfrentam dificuldades para concluir estudos-piloto, frequentemente realizados com recursos escassos e amostras pequenas. Este estudo propõe uma análise crítica sobre experiências com a evasão de participantes em quatro pesquisas que avaliaram programas de intervenção, conduzidas pelos autores do presente artigo, à luz da literatura recente. A análise das experiências revelou percentuais de $40 \%$ a $80 \%$ de abandono após o recrutamento ou início da intervenção. Entre os motivos elencados para justificar o abandono estão: o desinteresse, a discordância entre os cônjuges sobre a adesão, a distância entre residência e local da intervenção e a incompatibilidade de horários. Conclui-se que a maior aproximação entre os pesquisadores e a comunidade pode resultar em aumento de confiança e adesão aos estudos, o que pode ampliar o potencial de inserção social e divulgação científica das pesquisas realizadas com essa população.
\end{abstract}

Palavras-chave: Intervenções psicológicas, Evasão, Pais, Crianças, Adolescentes.

\section{Dropout in Psychological Interventions with Parents of Children and Adolescents: Experience Report}

\begin{abstract}
Dropouts in research evaluating the effects of interventions with parents of children and adolescents has been less investigated by Brazilian researchers, who face difficulties to complete pilot studies, often carried out with scarce resources and small samples. This study proposes a critical analysis of experiences with participant's dropouts in four pieces of research that evaluated intervention programs delivered by the authors of this paper, in light of recent literature. Analysis of the experiences revealed percentages between $40 \%$ and $80 \%$ of dropouts after the recruitment or the beginning of intervention. Among the reasons listed to justify the dropout are: lack of interest, the disagreement between the spouses related to the agreement, the distance between home and interventions' venue and mismatch of schedules. We conclude that a closer relationship between researchers and the community can result in an increase of trust and retention, which can expand the potential of social insertion and scientific dissemination of research conducted with this population.
\end{abstract}

Keywords: Psychological interventions, Dropouts, Parents, Children, Adolescents. 


\title{
Evasión en Intervenciones Psicológicas con Padres de Niños y Adolescentes: Informe de Experiencia
}

\begin{abstract}
Resumen: La evasión de participantes en investigaciones que evalúan efectos de intervenciones con padres de niños y adolescentes ha sido poco explorada por los investigadores brasileños, que enfrentan dificultades para concluir estudios piloto, frecuentemente realizados con recursos escasos y muestras pequeñas. Este estudio propone un análisis crítico sobre experiencias con la evasión de participantes en cuatro investigaciones que evaluaron programas de intervención, conducidas por los autores del presente artículo, a la luz de la literatura reciente. El análisis de las experiencias reveló porcentuales entre $40 \%$ y $80 \%$ de abandono después del reclutamiento o inicio de la intervención Entre los motivos enumerados para justificar el abandono están el desinterés, el desacuerdo entre los cónyuges sobre la adhesión, la distancia entre la residencia y el lugar de la intervención y la incompatibilidad de horarios. Se concluye que la mayor aproximación entre los investigadores y la comunidad puede resultar en un aumento de confianza y adhesión a los estudios, lo que puede ampliar el potencial de inserción social y divulgación científica de las investigaciones realizadas con esta población.
\end{abstract}

Palabras clave: Intervenciones psicológicas, Evasión, Padres, Niños, Adolescentes.

\section{Introdução}

A literatura tem sinalizado a necessidade de investigações de caráter interventivo, que estimulem a inter-relação entre a pesquisa e a prática, com o objetivo de desenvolver ações voltadas à prevenção e promoção de saúde junto a indivíduos e comunidades (Mencarelli, Aiello, \& Vaisberg, 2012; Miyazaki, Domingos, Valério, Santos, \& Rosa, 2002). Nesse sentido, a pesquisa que implica propostas de intervenção vem gradualmente tornando-se uma parceria importante entre a universidade e algumas instituições, oferecendo a possibilidade de contribuir para a formação de profissionais e, ao mesmo tempo, atender demandas de comunidades mais vulneráveis, nas quais os serviços são insuficientes ou carecem de profissionais (Szymanski \& Cury, 2004). De modo geral, essas intervenções fornecem informações que visam ao desenvolvimento de habilidades a serem incorporadas à rotina diária dos usuários por serem benéficas a sua saúde física ou mental (Friberg \& Scherman, 2005; Brasil, 2008; Straub, 2014). Além disso, por estarem vinculadas à pesquisa, há preocupação com sua validação, havendo avaliação rigorosa dos seus resultados.

No caso específico de programas de intervenção com mães e pais, os objetivos costumam estar relacionados a prevenir ou minimizar os efeitos de experiências adversas sobre o desenvolvimento de crianças e adolescentes, por meio de mudanças comportamentais dos pais (Folger et al., 2016; Weeland et al., 2017). Adicionalmente, as intervenções costumam focalizar o bem-estar e a satisfação em tarefas relacionadas à parentalidade (Löfgren, Petersen, Nilsson, Ghazinour, \& Hägglöf, 2017), como, por exemplo, disciplinar ou ensinar hábitos de saúde e higiene à criança.

Contudo, no Brasil, desenvolver pesquisas que envolvam intervenção, é, muitas vezes, desafiador devido às dificuldades que podem prejudicar a sua implementação e avaliação. Dentre essas dificuldades, destaca-se $o$ abandono dos programas de intervenção por parte dos participantes, que reflete um dos principais problemas relacionados à adesão. O conceito de adesão refere-se ao comportamento de um indivíduo de aceitar a proposta e participar ativamente do processo dinâmico e multifatorial relacionado ao cuidado de si ou de sua família, que envolve corresponsabilidade e parceria entre o participante e o pesquisador (Friberg \& Scherman, 2005; Straub, 2014). O abandono ou evasão seria um indicador extremo da não adesão, e se refere ao comportamento de deixar de participar das consultas ou sessões relativas à intervenção proposta. Em outras palavras, consiste na partida prematura dos pais desses serviços (McCurdy \& Daro, 2001).

Alguns estudos sugerem que os fenômenos da evasão e da baixa adesão a programas de atenção 
e suporte familiar só poderão ser claramente compreendidos pelo conhecimento das variáveis que os afetam em múltiplos níveis, tais como características individuais, atributos do facilitador, características do programa e do contexto da comunidade ou bairro atendido (Folger et al., 2016; Ingoldsby, 2010; McCurdy \& Daro, 2001). Em cada um desses níveis, muitos fatores podem ser identificados em distintas fases da intervenção, como no recrutamento, engajamento e retenção de participantes. Alguns estudos têm investigado tais aspectos para compreender o abandono de intervenções psicológicas com pais de crianças e adolescentes, como será abordado a seguir.

Entre as características individuais, mais do que a intenção de usar determinado serviço, é preciso verificar se os participantes estão preparados para esse tipo intervenção, o que requer o reconhecimento dos riscos e prejuízos para o desenvolvimento dos filhos e dos benefícios que o serviço pode trazer para minimizá-los (McCurdy \& Daro, 2001). Parte dos motivos pode estar relacionada à falta de percepção da necessidade da intervenção, assim como ao fato de os pais julgarem que os benefícios emergem lentamente (Ingoldsby, 2010), o que leva ao abandono ou à pouca motivação para persistir até o final dos programas. Porém, a consciência acerca desses aspectos não é suficiente para garantir a adesão, pois os pais podem não estar prontos para a mudança, ou seja, para as ações com que terão que se comprometer para melhorar a qualidade da relação com os filhos e promover seu desenvolvimento (Smith et al., 2015), o que também os faz desistirem de participar das intervenções.

O abandono das intervenções ainda pode ser associado a alguns sentimentos tais como o medo ou desconfiança em relação aos programas, ou em relação à estigmatização social que os indivíduos podem sofrer ao recorrerem a esse tipo de serviço, o que poderia ser compreendido pelo entorno social como um sinal de incompetência nos cuidados com os filhos (Koerting et al., 2013). Outros aspectos referidos pelos participantes como motivos para o abandono das intervenções são o horário e a carga horária de trabalho, assim como a falta de tempo disponível para os encontros (Koerting et al., 2013; Mytton, Ingram, Manns, \& Thomas, 2014). Além desses fatores, a concordância de ambos os pais com a participação na intervenção também aparece como um fator importante (McCurdy \& Daro, 2001).
No tocante às características do facilitador, destacam-se a consciência e a sensibilidade à história e ao contexto em que a família vive, além de sua capacidade de comunicação efetiva com os participantes (Flores et al., 2017; Koerting et al., 2013; McCurdy \& Daro, 2001). Outros aspectos relevantes são o número de casos acompanhados por facilitador, que não deve ser excessivo, e a qualidade do treinamento e supervisão que ele recebe (Mytton et al., 2014).

Segundo McCurdy e Daro (2001), no que diz respeito ao próprio programa, o tempo decorrido entre o recrutamento e o início da intervenção, bem como o uso de incentivos materiais podem afetar o engajamento dos pais. Portanto, sugere-se intervenções ágeis e breves em relação ao tempo de duração (Cia, Barhamm, \& Fontaine, 2010; Lim, Stormshak, \& Dishion, 2005; Rolfsen \& Martinez, 2008; Smith et al., 2015; Stormshak et al., 2011) e a disponibilização aos seus participantes de alimentação no local dos encontros e de auxílio financeiro para o transporte (Havighurst \& Harley, 2010; Marinho, 2005). Além dessas variáveis, o fato de o programa ter início em um período sensível para os participantes, como a fase pré-natal, por exemplo, poderia favorecer o engajamento, pois os pais estariam mais dispostos a receber auxílio e aconselhamento nesta etapa. Por fim, destaca-se a importância da supervisão continuada aos facilitadores e o financiamento estável dos programas para a garantia de sua qualidade (McCurdy \& Daro, 2001).

Quanto às características do bairro ou comunidade onde a família reside, é preciso considerar o impacto que a cultura local pode produzir sobre a percepção dos pais a respeito de propostas de intervenção relativas à parentalidade. Quando os vizinhos, amigos ou lideranças do bairro apoiam a iniciativa, é mais provável que os pais queiram se engajar. Por outro lado, se há na comunidade uma tendência a compreender a criação dos filhos como uma questão privada e não passível de interferência por parte de agentes externos, é pouco provável que tais propostas recebam suporte do grupo social (McCurdy \& Daro, 2001). Ainda é preciso considerar características como a violência urbana e outras circunstâncias de instabilidade vigentes na comunidade, que podem fazer com que os participantes tenham poucas expectativas em relação aos serviços ali oferecidos, ou mesmo que se sintam inseguros 
para receber visitas domiciliares ou frequentar locais públicos da vizinhança (Folger et al., 2016).

Considerando os motivos relacionados ao abandono das intervenções que foram discutidos, existem alternativas que podem ser exploradas no planejamento, divulgação e implementação das intervenções, com o objetivo de aumentar a adesão de pais. Contudo, ainda se faz necessário refletir sobre estratégias exitosas para aumentar a capacidade de recrutamento e a retenção de participantes, uma vez que há poucas evidências acerca desta questão.

Ao planejar estudos de avaliação de intervenção o pesquisador deve, inicialmente, refletir sobre a posição que assume frente ao campo de pesquisa e as relações que estabelece com os participantes, bem como sobre os efeitos produzidos pela sua participação (Paulon, 2005). Tal cuidado se justifica porque a relação entre pesquisadores e participantes é determinante para o sucesso ou o fracasso de estudos de intervenção (Brookman-Frazee et al., 2016; Flores et al., 2017; Joseph, Keller, \& Ainsworth, 2016; Tramm, Daws, \& Schadewaldt, 2013). Autores têm enfatizado a importância de desenvolver uma relação de confiança e respeito com potenciais participantes (Flores et al., 2017), e, para tanto, recomendam manter uma comunicação direta, respondendo dúvidas com prontidão e cortesia, utilizando, com cada participante, a forma de comunicação que preferirem, como, por exemplo, e-mails, mensagens de texto ou telefonemas (Joseph et al., 2016; Marinho, 2005; Pozzobon, Falcke, \& Marin, 2018).

Várias pesquisas têm destacado a importância de adaptar o serviço de forma que ele se alinhe melhor às demandas dos indivíduos que estão sendo atendidos (Garcia-Huidobro et al., 2016; Havighurst, \& Harley, 2010; Ingoldsby, 2010; Koerting et al., 2013; Mytton et al., 2014; Smith et al., 2015; Szymanski \& Cury, 2004). Uma forte concordância entre os objetivos e expectativas dos pais e aqueles mantidos pelo programa de intervenção e, portanto, pelo facilitador, parecem favorecer o engajamento dos participantes. Nesse sentido, manter certa flexibilidade para contemplar os objetivos dos pais, parece ser uma estratégia promissora para evitar a evasão (McCurdy \& Daro, 2001).

Outra estratégia recomendada é a formação de parcerias com as comunidades onde acontecem os processos de recrutamento. Folger et al. (2016) avaliaram os efeitos de uma abordagem de fortalecimento da retenção de participantes em um programa de visitas domiciliares para famílias nos três primeiros anos de vida da criança. As estratégias de fortalecimento incluíram a formação de um comitê da comunidade, que discutia o programa com os provedores, com vistas a melhor adaptá-lo às demandas da população atendida, e apoiava estratégias de divulgação e recrutamento de participantes. A análise dos efeitos dessa e das outras estratégias utilizadas revelaram um aumento da retenção de participantes no programa de intervenção.

Nessa mesma perspectiva, o estudo de Brookman-Frazee et al. (2016) buscou caracterizar o uso da parceria pesquisa-comunidade em intervenções baseadas em evidências em serviços comunitários direcionados a crianças. Os resultados mostraram que os pesquisadores perceberam ganhos em termos de relevância e adaptabilidade do programa às demandas dos participantes, assim como uma melhoria nos serviços prestados e nos resultados obtidos. Os parceiros das comunidades também reconheceram a melhora da qualidade dos serviços e referiram resultados superiores aos habituais derivados da parceria pesquisa-comunidade. De modo semelhante, Joseph et al. (2016) recomendaram essa estratégia, que, segundo eles, pode ser utilizada mesmo em estudos pilotos ou que possuam recursos financeiros e humanos limitados.

Outra indicação importante se refere ao uso de estratégias para aumentar o conhecimento dos indivíduos sobre o problema focalizado pela intervenção a fim de sensibilizá-los (Cunha, 2015; Faustino \& Seidl, 2010; Pupo, 2015; Santos, Nasser, Basso, \& Paiva, 2013), como, por exemplo, a distribuição de material informativo em locais estratégicos (Joseph et al., 2016). Outro tipo de prática que pode ser utilizado com esse objetivo, é enfatizar para os participantes a importância do estudo para que, a partir de seus resultados, outras crianças e adolescentes possam vir a ser beneficiados (Flores et al., 2017).

Por fim, sugere-se, ainda, a criação de grupos ou encontros de "recuperação" ou "reforço" para atender aqueles que foram impedidos, por alguma razão, de comparecer à intervenção nas datas previstas (Folger et al., 2016). Nesse sentido, Flores et al. (2017) sugerem discutir os casos que apresentaram baixa adesão ou dificuldades de comunicação, com o objetivo de definir planos de ação que incluam telefonemas em 
diferentes horários do dia, tentativas de contato com parentes e amigos e visitas domiciliares.

Como visto, há um corpo de evidências consistente a respeito de fatores que podem explicar a evasão de participantes e estratégias que podem ser empregadas em estudos que avaliam intervenções com pais de crianças e adolescentes, principalmente em contextos internacionais. Contudo, os estudos realizados no Brasil a esse respeito, tais como os de Cia et al. (2010), Rolfsen e Martinez (2008) e Coelho e Murta (2007), são escassos, embora os pesquisadores com frequência se deparem com esse tipo de problema em pesquisas que avaliam os efeitos de intervenções com amostras pequenas e recursos humanos e financeiros limitados, características comuns à pesquisa nacional em Ciências Humanas e Sociais Aplicadas. Nesse sentido, analisar experiências de pesquisadores brasileiros com a evasão de participantes confrontando-as com os achados de estudos que investigaram ou teorizaram de modo mais aprofundado e sistemático sobre esse problema, pode ser um passo importante para avançar na sua compreensão. Assim, este estudo propõe uma análise crítica sobre experiências com a evasão de participantes em quatro pesquisas que avaliaram programas de intervenção, conduzidas pelos autores do presente artigo, à luz da literatura recente sobre o fenômeno.

\section{Método}

Neste estudo, será relatada a experiência com a evasão de participantes em quatro investigações brasileiras que avaliaram os efeitos de programas de intervenção psicológica com pais de crianças e adolescentes. O Quadro comparativo apresenta as principais características dos quatro estudos. Os estudos de intervenção com pais que serão examinados foram desenvolvidos em uma cidade do Nordeste (três programas) e em uma cidade do Sul (um programa) do Brasil. Três deles foram realizados na modalidade grupal e um deles consistiu em um programa de visitas domiciliares. Todos receberam financiamento de agências de fomento à pesquisa, mas tratavam-se de estudos-piloto, que contaram com recursos humanos e financeiros limitados. Em dois desses estudos, os recursos se resumiram à bolsa de mestrado de um dos facilitadores que conduziu a intervenção e ao apoio de um bolsista de iniciação científica nas atividades de um dos grupos.

\section{Resultados}

O primeiro estudo, cuja intervenção testada foi chamada de Programa de Responsividade Materna (PRM), visou a aumentar os níveis de responsividade materna e favorecer o desenvolvimento socioemocional e cognitivo da criança. A intervenção se desenvolveu em oito visitas domiciliares entre o terceiro e o décimo mês de vida do bebê. O programa teve como base alguns dos segmentos do Programa de Apoio Psicológico P/Materno-Infantil@ (PAPMI), desenvolvido na Espanha, e sua adaptação para a Irlanda (Parent-Child Psychological Support Program ${ }^{\mathrm{TM}}$ - PCPS; Cerezo, Dasi, \& Ruiz, 2013). Das 31 díades inicialmente recrutadas em unidades básicas de família e convidadas a participar do grupo intervenção do estudo que avaliou os efeitos do programa, nove abandonaram o programa. Duas mães deixaram de participar antes mesmo de a intervenção ser iniciada, sendo que uma alegou que seu marido não havia concordado com sua participação e, a outra, que não gostaria de ser filmada. Sete participantes abandonaram a intervenção enquanto ela estava em andamento. As justificativas das participantes que, por decisão própria, abandonaram a intervenção, foram a mudança de residência para um bairro distante (duas participantes) e não ter mais interesse na intervenção (uma participante). As demais (quatro participantes) foram desligadas do programa devido a dificuldades recorrentes de contato, somadas a inúmeras remarcações de visitas devido à ausência da participante em seu domicílio no dia da visita, ou pela falta de espaço para realizar a intervenção em virtude do grande número de pessoas presentes na casa.

O PRM empregou algumas estratégias para aumentar o número de recrutamentos e para evitar a desistência ao longo das visitas. Na fase de recrutamento, a equipe distribuiu panfletos com informações sobre a pesquisa nas ruas da comunidade e esteve presente nas unidades básicas de saúde conversando com os profissionais e com mães que frequentavam os serviços para divulgar o estudo. Além dessas estratégias de divulgação, a pesquisadora responsável pelo estudo apresentou o projeto e a equipe em reuniões realizadas nas unidades básicas e também divulgou o estudo na rádio de uma das duas comunidades participantes. Na primeira visita a cada díade, as facilitadoras informavam às mães que a intervenção era um tipo de serviço psicológico que a universidade estava prestando àquela comunidade e que contavam com o 
Quadro.

Quadro comparativo dos estudos dos programas de intervenção avaliados.

\begin{tabular}{|c|c|c|c|c|}
\hline & $\begin{array}{c}\text { Estudo 1 } \\
\text { Programa de } \\
\text { Responsividade } \\
\text { Materna (PRM) }\end{array}$ & $\begin{array}{c}\text { Estudo } 2 \\
\text { Programa de Práticas } \\
\text { de Socialização } \\
\text { Emocional (PPSE) }\end{array}$ & $\begin{array}{c}\text { Estudo } 3 \\
\text { Grupo de Práticas } \\
\text { Parentais (GPP) }\end{array}$ & \begin{tabular}{|c|} 
Estudo 4 \\
Intervenção com \\
Famílias de Alunos \\
com Dificuldades \\
Escolares (IFADE)
\end{tabular} \\
\hline $\begin{array}{l}\text { Objetivo da } \\
\text { intervenção }\end{array}$ & $\begin{array}{l}\text { Aumentar os níveis } \\
\text { de responsividade } \\
\text { materna e favorecer } \\
\text { o desenvolvimento } \\
\text { socioemocional e } \\
\text { cognitivo da criança }\end{array}$ & $\begin{array}{l}\text { Promover as práticas } \\
\text { de socialização } \\
\text { emocional de mães } \\
\text { diante de emoções } \\
\text { negativas dos filhos } \\
\text { e de reduzir os } \\
\text { escores de problemas } \\
\text { internalizantes das } \\
\text { crianças }\end{array}$ & $\begin{array}{l}\text { Aprimorar as práticas } \\
\text { de socialização dos } \\
\text { pais e reduzir os } \\
\text { escores de problemas } \\
\text { internalizantes de } \\
\text { crianças em direção a } \\
\text { valores não clínicos }\end{array}$ & $\begin{array}{c}\text { Promover o } \\
\text { suporte familiar } \\
\text { para melhoria } \\
\text { do desempenho } \\
\text { de adolescentes } \\
\text { com histórico } \\
\text { de dificuldades } \\
\text { escolares }\end{array}$ \\
\hline $\begin{array}{l}\text { Formação dos } \\
\text { facilitadores }\end{array}$ & $\begin{array}{c}\text { Graduandas em } \\
\text { Psicologia treinadas } \\
\text { e supervisionadas } \\
\text { pela pesquisadora } \\
\text { responsável } \\
\text { (graduada, mestre } \\
\text { e doutora em } \\
\text { Psicologia) }\end{array}$ & $\begin{array}{l}\text { Graduada e mestre } \\
\text { em Psicologia } \\
\text { supervisionada } \\
\text { pela pesquisadora } \\
\text { responsável }\end{array}$ & $\begin{array}{c}\text { Graduado e } \\
\text { mestrando } \\
\text { em Psicologia } \\
\text { supervisionado } \\
\text { pela pesquisadora } \\
\text { responsável }\end{array}$ & $\begin{array}{c}\text { Graduada e } \\
\text { mestranda em } \\
\text { Psicologia Clínica } \\
\text { supervisionada } \\
\text { pela pesquisadora } \\
\text { responsável }\end{array}$ \\
\hline $\begin{array}{l}\text { Duração da } \\
\text { intervenção }\end{array}$ & 8 meses & 2 meses & 3 meses & 4 meses \\
\hline Ano/Região & 2013-2016/NE & 2016/NE & 2012/NE & $2016 / \mathrm{S}$ \\
\hline $\begin{array}{l}\text { Modalidade/ } \\
\text { técnicas } \\
\text { empregadas }\end{array}$ & $\begin{array}{l}\text { Visitas domiciliares/ } \\
\text { Vídeo feedback e } \\
\text { modelação. }\end{array}$ & $\begin{array}{l}\text { Grupal /Ensaios } \\
\text { comportamentais, } \\
\text { análise de } \\
\text { contingências com } \\
\text { base nos relatos } \\
\text { maternos, análises } \\
\text { de cenas de filmes e } \\
\text { imagens, atividades } \\
\text { de psicoeducação, } \\
\text { entre outras }\end{array}$ & $\begin{array}{l}\text { Grupal/ Observação } \\
\text { e registro de } \\
\text { comportamento, } \\
\text { análise de } \\
\text { contingências, ensaio } \\
\text { comportamental, } \\
\text { oferecimento de } \\
\text { modelos, dinâmicas } \\
\text { de grupo e tarefas de } \\
\text { casa }\end{array}$ & $\begin{array}{c}\text { Grupal/ } \\
\text { Externalização } \\
\text { do problema } \\
\text { através de recursos } \\
\text { expressivos } \\
\text { (desenho, escrita } \\
\text { criativa, argila e } \\
\text { sucatas) }\end{array}$ \\
\hline $\begin{array}{l}\text { Recursos } \\
\text { financeiros }\end{array}$ & $\begin{array}{l}\text { Apoio financeiro a } \\
\text { projeto de pesquisa } \\
\text { (CNPq) }\end{array}$ & $\begin{array}{c}\text { Apoio financeiro a } \\
\text { projeto de pesquisa } \\
(\mathrm{CNPq})\end{array}$ & $\begin{array}{c}\text { Bolsa de mestrado } \\
\text { (CNPq) }\end{array}$ & $\begin{array}{c}\text { Apoio financeiro a } \\
\text { projeto de pesquisa } \\
\text { (CNPq) }\end{array}$ \\
\hline $\begin{array}{l}\text { Núcleo de } \\
\text { recrutamento }\end{array}$ & $\begin{array}{c}\text { Unidades Básicas de } \\
\text { Saúde }\end{array}$ & Escolas & Escolas & Escolas \\
\hline $\begin{array}{l}\text { Contato para } \\
\text { recrutamento }\end{array}$ & $\begin{array}{c}\text { Panfletos, encontros } \\
\text { nas Unidades Básicas } \\
\text { de Saúde e rádio } \\
\text { comunitária }\end{array}$ & $\begin{array}{l}\text { Encontros na escola } \\
\text { com a facilitadora }\end{array}$ & $\begin{array}{l}\text { Encontros na escola } \\
\text { com o facilitador }\end{array}$ & $\begin{array}{c}\text { Via coordenação de } \\
\text { escolas e reuniões } \\
\text { com convidados e } \\
\text { facilitadora }\end{array}$ \\
\hline
\end{tabular}

NE: Nordeste; S: Sul; CNPq: Conselho Nacional de Desenvolvimento Científico e Tecnológico.

seu compromisso de comparecer às visitas nas datas e horários marcados para possibilitar que um bom trabalho fosse realizado e para que outras crianças pudessem ser beneficiadas pelos resultados da pes- quisa. Nessa mesma ocasião, cada mãe recebia um ímã em que constava um calendário com a previsão de oito visitas domiciliares entre o terceiro e o décimo mês de vida do bebê e espaço para o preenchimento 
das datas em que as visitas aconteceriam. A cada visita realizada, a facilitadora solicitava o calendário para registrar a data da visita seguinte, após estabelecer o melhor dia e horário em acordo com a mãe. A facilitadora alertava a participante quanto à importância de deixar o ímã afixado em local visível para não esquecer a data da visita. Adicionalmente, no dia anterior à data marcada, a facilitadora telefonava para a participante confirmando o encontro.

O segundo estudo, cuja intervenção testada foi o Programa de Práticas de Socialização Emocional (PPSE), foi realizado em uma escola com o objetivo de promover as práticas de socialização emocional de mães diante de emoções negativas dos filhos e de reduzir os escores de problemas internalizantes das crianças. A intervenção aconteceu em oito sessões grupais e foi fundamentada no programa Tuning in to Kids ${ }^{\mathrm{TM}}$ (Havighurst \& Harley, 2010). Foram recrutadas mães de crianças com três a cinco anos que apresentavam indicadores limítrofes e clínicos de problemas internalizantes. No total, 28 mães foram convidadas a participar do programa de intervenção, contudo, nove informaram, no momento do convite, que não poderiam participar devido a: dificuldade de conciliar o horário do grupo com o do trabalho (três mães) ou com atividades que desempenhavam na família, como o cuidado de pais idosos (uma mãe) e dificuldades relacionadas ao local de realização do programa (cinco mães). Dezenove mães confirmaram interesse em participar do estudo, porém, na primeira sessão, apenas seis estiveram presentes e uma havia informado previamente que só conseguiria começar a participar a partir da segunda sessão. Além disso, uma das mães informou que não poderia participar devido ao dia escolhido pela maioria das mães para a realização do grupo que não coincidia com o seu dia de folga do trabalho. Entre a primeira e a segunda sessão, foi feita uma tentativa de contato com as mães que não compareceram e, aquelas com quem foi possível o contato informaram dificuldades pessoais para participar (cinco mães) ou confirmaram que iriam à segunda sessão (duas mães). A segunda sessão contou com sete participantes. Contudo, três mães abandonaram o programa durante o período da sua realização e apenas quatro o concluíram. Somente duas apresentaram justificativas para a descontinuidade: novo emprego (uma mãe) e problemas familiares que estavam lhe tomando tempo excessivo (uma mãe).
O PPSE adotou algumas estratégias para evitar o abandono por parte dos participantes. Na primeira sessão, foram estabelecidas com as mães algumas regras para o funcionamento do grupo. As regras incluíam o compromisso de participar de todas as sessões, visto que em cada sessão um tema diferente seria abordado, e, portanto, faltas poderiam prejudicar o andamento do grupo e o aproveitamento individual. Outra estratégia adotada envolveu o contato por telefone com as mães durante a semana, especialmente no dia anterior à sessão, com o objetivo de motivá-las para a reunião do dia seguinte. As facilitadoras do programa também enviavam mensagens de texto via aplicativo de troca de mensagens, lembrando às mães sobre a próxima sessão. As participantes costumavam confirmar a participação, mas caso informassem alguma dificuldade era realizado contato telefônico para mais esclarecimentos. Em todas as sessões, também foi oferecido um lanche que funcionava tanto como um momento de pausa nas atividades como para favorecer a confraternização e a aproximação entre as mães e as facilitadoras. Além disso, em algumas sessões, as mães foram convidadas a avaliar o andamento do programa e aspectos que poderiam ser enfatizados ou melhorados nas sessões seguintes.

O terceiro estudo, que avaliou o programa chamado Grupo de Práticas Parentais (GPP) (Oliveira \& Alvarenga, 2015), foi realizado em três escolas com o objetivo de aprimorar as práticas de socialização dos pais e reduzir os escores de problemas internalizantes de crianças, que apresentavam escores clínicos nesse perfil, em direção a valores não clínicos. O GPP é um programa de intervenção grupal de oito sessões, elaborado com base nas propostas de orientação a pais de Bolsoni-Silva, Carrara e Marturano (2008) e Coelho e Murta (2007). O grupo foi oferecido a mães e pais de crianças de três a seis anos, com escores limítrofes ou clínicos para problemas internalizantes. O convite foi feito para 64 famílias, das quais 14 participaram das primeiras sessões em três grupos realizados em diferentes escolas. Dos 14 pais e mães que iniciaram, apenas sete concluíram os encontros. Os participantes relataram motivos associados à dificuldade de conciliar o horário dos encontros com as atividades cotidianas (seis pais) e à falta de interesse nas atividades (uma mãe).

Uma das estratégias utilizadas no GPP para evitar a perda de participantes foi a negociação das regras 
Tabela 1

Porcentagem de evasão nos quatro estudos apresentados.

\begin{tabular}{lccc}
\hline Estudo & Amostra inicial & Amostra final & Evasão (\%) \\
\hline 1 & 31 díades & 18 díades & 41,94 \\
2 & 19 mães & 4 mães & 78,95 \\
3 & 14 pais & 7 pais & 50,00 \\
4 & 24 famílias & 9 famílias & 62,50 \\
\hline
\end{tabular}

de funcionamento. Dentre as regras, a assiduidade aos encontros foi enfatizada como uma das principais características associadas ao bom andamento da intervenção. Além disso, cada sessão foi dividida em dois momentos intercalados por uma confraternização com lanche entre os participantes e o facilitador. Outra estratégia importante foi a oferta de grupos em diferentes escolas e horários para evitar que a distância entre a residência e o local da intervenção ocasionasse a desistência dos pais.

Por fim, o quarto estudo, decorrido em seis encontros, avaliou o programa intitulado Intervenção com Famílias de Alunos com Dificuldades Escolares (IFADE). A pesquisa objetivou investigar a eficácia de uma intervenção que visava promover o suporte familiar para melhoria do desempenho de adolescentes com histórico de dificuldades escolares. Apesar de a proposta ter contado com grande apoio e colaboração das equipes diretivas das duas escolas participantes, que se envolveram ativamente na fase dos convites e tentativa de conscientização dos pais sobre a importância do programa, também houve um número importante de desistências. Das 166 famílias convidadas a participarem do programa, por terem seus filhos adolescentes apresentado baixo desempenho escolar e reprovação nos últimos dois anos, somente 24 participaram da reunião de adesão. Destas, oito evadiram após o primeiro encontro, seis no segundo e uma no terceiro, sendo que somente nove famílias concluíram o programa. Várias justificativas foram elencadas, como esquecimentos, distância entre o trabalho e o local dos encontros, incompatibilidade de horários, assim como a presença de filhos pequenos e parentes doentes que necessitavam de atenção. Nesse sentido, foi observado que algumas famílias pareciam se importar menos com os problemas dos filhos em idade escolar e mais com as questões destacadas, que julgavam mais urgentes. As greves dos professo- res municipais, que reivindicavam melhores salários na ocasião, também colaboraram para que os dias e horários dos encontros tivessem que ser mudados, $o$ que provocou a sensação de desorganização entre os participantes. Além disso, o período de desenvolvimento da intervenção foi marcado por intempéries que assolaram a cidade à época do estudo.

As estratégias utilizadas para prevenir o abandono foram: desenvolver uma intervenção breve, com seis encontros, estabelecer um bom rapport desde os primeiros contatos, acessar constantemente as famílias e ofertar alimento durante os encontros. Ainda, devido às sucessivas faltas, foi criado um sistema de recuperação em horário alternativo.

Constata-se, portanto, que, mesmo diante das várias tentativas de prevenção à evasão recomendadas pela literatura e adotadas pelos estudos apresentados, evidenciaram-se importantes índices de abandono, como mostra a Tabela 1. Os percentuais variaram entre $42 \%$ e $79 \%$, indicando que cerca da metade dos participantes recrutados para as intervenções abandonaram as pesquisas.

\section{Discussão}

Os estudos descritos abordam temáticas que perpassam o desenvolvimento infantil e adolescente, com vistas a promover contextos de interação familiar mais funcionais. Contudo, há importantes dificuldades relacionadas à condução de programas de intervenção, especialmente associadas à evasão dos participantes, o que exige das equipes grande mobilização e criatividade. Os dados numéricos da evasão nos quatro estudos mostram que cerca da metade ou mais dos participantes abandonaram as pesquisas após o recrutamento ou início da intervenção.

Os múltiplos níveis de análise e as variáveis relacionadas a cada um deles, apontados por alguns dos estudos sobre evasão de participantes que foram revisados (Folger et al., 2016; Ingoldsby, 
2010; McCurdy \& Daro, 2001) puderam ser evidenciados no conjunto dos quatro estudos. No que se refere ao nível das características individuais, vários foram os motivos elencados para justificar a evasão, muitos deles já relatados em estudos anteriores, tais como esquecimento ou desinteresse na participação, discordância entre os cônjuges sobre a adesão (McCurdy \& Daro, 2001), distância entre residência e local da intervenção, incompatibilidade de horários ou falta de tempo (Koerting et al., 2013; Mytton et al., 2014), e dificuldade em conciliar a intervenção com outras tarefas e demandas.

Motivos como o esquecimento, o desinteresse e a falta de concordância de ambos os cônjuges podem indicar a necessidade de fortalecer e aprimorar estratégias de divulgação da pesquisa. Sobretudo, é necessário encontrar uma linguagem que seja, não apenas compreensível e familiar para os participantes, mas que também tenha o potencial de sensibilizá-los para a importância dos pais no processo de desenvolvimento dos filhos e para os benefícios que a intervenção pode produzir. A linguagem acadêmica a que os pesquisadores estão habituados, e que faz parte da sua rotina de trabalho diária, é um empecilho a ser considerado com mais atenção. Seriam necessários, além do processo usual de interação com a comunidade para a inserção dos pesquisadores e divulgação do estudo, encontros que permitissem um diálogo continuado com potenciais participantes sobre o tema da pesquisa. Esse tipo de interação poderia facilitar ajustes na linguagem dos pesquisadores assim como permitiria o conhecimento e maior aproximação da cultura local. Acredita-se que o investimento nesse tipo de estratégia tanto fortaleceria o recrutamento quanto aumentaria o engajamento e a retenção de participantes.

No que se refere a dificuldades de horários e de conciliar a intervenção com outras tarefas, além da distância do local da intervenção, a modalidade de visitas domiciliares pode ser uma alternativa interessante. Entre os quatro estudos, o primeiro, que adotou essa estratégia, foi aquele que teve o menor percentual de abandono. É possível que as visitas domiciliares, portanto, facilitem a composição entre os compromissos de rotina, as dificuldades de deslocamento e tempo e a participação na pesquisa, aumentando a retenção de participantes.

Além disso, mesmo que não seja possível identificar nos motivos alegados se os participantes estavam ou não preparados para o tipo intervenção proposto, conforme McCurdy e Daro (2001) sugerem, é plausível supor que, mesmo após os esclarecimentos por parte da equipe de profissionais, podem não ter ficado claros os benefícios que as intervenções poderiam oferecer a suas famílias (Ingoldsby, 2010). Tal questão pode ser justificada, em parte, devido ao baixo nível de escolaridade apresentado pelos participantes, que podem ter dificuldades para compreender as explicações e argumentos utilizados pelos pesquisadores na etapa de divulgação dos estudos e recrutamento da amostra. Nesse sentido, as estratégias que promovem maior contato e oportunidades de diálogo entre a população alvo da intervenção e os pesquisadores ou facilitadores, podem aumentar o número de participantes recrutados e retidos, assim como favorecer o engajamento na intervenção.

Embora os sentimentos de medo, desconfiança ou estigmatização em relação aos programas de intervenção não tenham sido identificados, conforme sugerem Koerting et al. (2013), constatou-se o sentimento de timidez ou vergonha entre alguns dos participantes. Também foi observado que alguns deles não conseguiam dimensionar o benefício que poderiam ter com a intervenção e pareciam se importar menos com os problemas dos filhos relativos à escola e mais com outras questões que julgavam prementes, como a atenção a filhos menores ou parentes doentes. Tais sentimentos podem ter dificultado o engajamento, atuando como um dos motivadores para a evasão. Iniciativas de divulgação científica da Psicologia na comunidade em geral, especialmente através de tecnologias digitais, podem contribuir para minimizar os efeitos desse tipo de estigma ao mostrar a aplicabilidade do conhecimento psicológico na vida cotidiana.

Quanto ao nível referente às características dos programas, todos se constituíram como breves, contando com seis a oito encontros, conforme sugere a literatura (Cia et al., 2010; Lim et al., 2005; Rolfsen \& Martinez, 2008; Smith et al., 2015; Stormshak et al., 2011). Também foram atendidos outros critérios, tais como o cuidado com reuniões prévias para convite e esclarecimentos, colocação de regras claras de funcionamento dos grupos, ampla divulgação no local das intervenções (Joseph et al., 2016), avaliação e reavaliação das atividades, oferta de alimento nos encontros (Havighurst \& Harley, 2010; Marinho, 2005), realização de grupos em mais de um local, facilitando o 
acesso, e de grupos de recuperação para faltas (Folger et al., 2016). Portanto, no que se refere a este nível de análise, os quatro programas implementaram um conjunto consistente de estratégias que contemplam grande parte dos aspectos apontados nos estudos revisados. Esses esforços certamente evitaram que a evasão fosse maior ou que o número de participantes recrutados fosse ainda menor.

No que diz respeito ao nível de análise referente às características do facilitador, todos eram pelo menos graduandos do curso de Psicologia e eram supervisionados por profissionais com maior nível de formação. Além disso, buscou-se aproximar a equipe e os participantes por meio do estabelecimento de um bom rapport, manutenção de proximidade e comunicação constante por meio de telefonemas e mensagens (Cia et al., 2010; Joseph et al., 2016; Lim et al., 2005; Marinho, 2005; Pozzobon et al., 2018; Rolfsen \& Martinez; 2008; Stormshak et al., 2011).É possível que um preparo mais específico do facilitador para adequar sua linguagem àquela tipicamente utilizada pela população-alvo da intervenção, somada a uma inserção continuada e estável dos grupos de pesquisa nas comunidades e escolas consigam fortalecer as estratégias comumente utilizadas, aumentando a captação e retenção de participantes.

Por fim, é necessário analisar o nível do contexto da comunidade ou bairro em que a intervenção foi oferecida. Todos os programas de intervenção apresentados foram ofertados de modo pontual em comunidades ainda não conhecidas pelos facilitadores e pesquisadores, o que pode ter tornado mais difícil o desenvolvimento de estratégias de recrutamento e retenção e a adaptação de cada etapa dos programas ao público-alvo. Nesse sentido, reitera-se a realização das pesquisas num modelo de parceria entre pesquisadores e comunidade, tal como preconizam Brookman-Frazee et al. (2016), para melhor adequar a intervenção às necessidades dos indivíduos que serão beneficiados, contando com o apoio da própria comunidade (Folger et al., 2016; Garcia-Huidobro et al., 2016; Ingoldsby, 2010; Joseph et al., 2016; Koerting et al., 2013; McCurdy \& Daro, 2001; Mytton et al., 2014; Smith et al., 2015; Szymanski \& Cury, 2004). Acredita-se que a maior aproximação entre os pesquisadores e a comunidade pode resultar em aumento de confiança e adesão aos estudos (Brookman-Frazee et al., 2016; Flores et al., 2017; Joseph, Keller, \& Ainsworth, 2016; Tramm, Daws, \&
Schadewaldt, 2013). Esse parece ser o nível menos atingido pelas estratégias adotadas pelos pesquisadores dos quatro estudos examinados, e possivelmente neste nível estão as variáveis das quais derivaram as causas mais relevantes da baixa retenção de participantes e do interesse reduzido dos pais pela participação nas intervenções.

\section{Considerações finais}

Os estudos que avaliam efeitos de intervenção têm se tornando mais frequentes no Brasil, mas ainda se faz necessário refletir sobre como vêm sendo conduzidos e as dificuldades encontradas, considerando as especificidades culturais do país. É comum enfatizar os dados obtidos e as principais conclusões, restringindo a discussão sobre suas limitações, especialmente no tocante às dificuldades implicadas no processo da pesquisa. Nesse sentido, relatos de experiência, como o presente, são necessários para que cada experiência seja tomada como base para as seguintes.

Como visto, foram examinados aspectos dos quatro estudos que correspondem aos diferentes níveis de análise sugeridos pela literatura revisada, e que contemplam as características dos participantes, dos programas, dos facilitadores e dos bairros ou contextos de aplicação das intervenções. Concluiu-se que estudos que investigam os efeitos de intervenções com pais de crianças e adolescentes devem investir em estratégias que: (a) possibilitem a adaptação da linguagem dos facilitadores e do próprio manual da intervenção às características da linguagem comumente utilizada pela população alvo do programa; e (b) permitam um diálogo continuado com potenciais participantes sobre o tema da pesquisa. Além dessas duas estratégias específicas, iniciativas mais amplas de divulgação científica da Psicologia na comunidade, especialmente através de tecnologias digitais, são necessárias para mostrar a aplicabilidade do conhecimento psicológico na vida cotidiana e reduzir o estigma que relaciona à Psicologia exclusivamente ao tratamento de transtornos mentais graves.

Este estudo possibilitou aproximar programas de intervenção realizados em contextos regionais diferentes do país, gerando o compartilhamento de ansiedades, sucessos e frustrações entre os pesquisadores, o que parece ser importante e necessário para avanços no sentido de ampliar o campo de visão e de atuação do psicólogo, aproximando pesquisa e prática no 
desenvolvimento e aprimoramento de ações de caráter preventivo junto às comunidades brasileiras e respeitando suas peculiaridades (Mencarelli et al., 2012; Miyazaki et al., 2002). Adicionalmente, acredita-se que a realização de estudos como este e a promoção de debates acerca dessa problemática podem ampliar o potencial de inserção social e divulgação científica das pesquisas realizadas no nosso país.

\section{Referências}

Bolsoni-Silva, A. T., Carrara, M. P., \& Marturano, E. M. (2008). Intervenção em grupo para pais: A que estar atento com relação à intervenção e às habilidades terapêuticas? In M. R. Cavalcante (Ed.), Análise do comportamento: avaliação e intervenção, (pp. 102-135). São Paulo, SP: Roca.

Brasil. Ministério da Saúde. (2008). Manual de adesão ao tratamento para pessoas vivendo com HIV e Aids. Brasília, DF: o autor. Recuperado de http://bvsms.saude.gov.br/bvs/publicacoes/manual_adesao_tratamento_hiv.pdf

Brookman-Frazee, L., Stahmer, A., Stadnick, N., Chlebowski, C., Herschell, A., \& Garland, A. (2016). Characterizing the use of research-community partnerships in studies of evidence-based interventions in children's community services. Administration and Policy in Mental Health and Mental Health Services Research, 43(1), 93-104. https://doi.org/10.1007/s10488-014-0622-9

Cerezo, M. A., Dasi, C., \& Ruiz, J. C. (2013). Supporting parenting of infants: Evaluating outcomes for parents and children in a community-based program. Evaluation and Program Planning, 37, 12-20. https://doi.org/10.1016/j. evalprogplan.2012.12.003

Cia, F., Barham, E. J., \& Fontaine, A. M. G. V. (2010). Impactos de uma intervenção com pais: O desempenho acadêmico e comportamento das crianças na escola. Psicologia: Reflexão e Crítica, 23(3), 533-543. https://doi. org/10.1590/S0102-79722010000300014

Coelho, M. V., \& Murta, S. G. (2007). Treinamento de pais em grupo: Um relato de experiência. Estudos de Psicologia (Campinas), 24(3), 333-341. https://doi.org/10.1590/S0103-166X2007000300005

Cunha, C. L. F. (2015). Projeto de intervenção para aumentar a adesão ao tratamento medicamentoso de pacientes hipertensos na área de abrangência da ESF Bela Vista em Ibapa - MG (Trabalho de conclusão de curso). Universidade Federal do Triangulo Mineiro, Ipatinga, MG, Brasil.

Faustino, Q. M., \& Seidl, E. M. F. (2010). Intervenção cognitivo-comportamental e adesão ao tratamento em pessoas com HIV/Aids. Psicologia: Teoria e Pesquisa, 26(1), 121-130. https://doi.org/10.1590/S010237722010000100014

Flores, G., Portillo, A., Lin, H., Walker, C., Fierro, M., Henry, M. et al. (2017). A successful approach to minimizing attrition in racial/ethnic minority, low-income populations. Contemporary Clinical Trials Communications, 5, 168-174. https://doi.org/10.1016/j.conctc.2017.01.009

Folger, A. T., Brentley, A. L., Goyal, N. K., Hall, E. S., Sa, T., Peugh, J. L. et al. (2016). Evaluation of a community-based approach to strengthen retention in early childhood home visiting. Prevention Science, 17(1), 52-61. https://doi.org/10.1007/s11121-015-0600-9

Friberg, F., \& Scherman, M. H. (2005). Can a teaching and learning perspective deepen understanding of the concept of compliance? A theoretical discussion. Scandinavian Journal of Caring Sciences, 19(3), 274-279. https://doi.org/10.1111/j.1471-6712.2005.00341.x

Garcia-Huidobro, D., Allen, M., Rosas-Lee, M., Maldonado, F., Gutierrez, L., Svetaz, M. V. et al. (2016). Understanding attendance in a community-based parenting intervention for immigrant latino families. Health Promotion Practice, 17(1), 57-69. https://doi.org/10.1177/1524839915582155

Havighurst, S. \& Harley, A. (2010). Tuning in to kids program manual. Melbourne: The University of Melbourne. (Trabalho original publicado em 2007).

Ingoldsby, E. M. (2010). Review of interventions to improve family engagement and retention in parent and child mental health programs. Journal of Child and Family Studies, 19(5), 629-645. https://doi.org/10.1007/s10826009-9350-2 
Joseph, R. P., Keller, C., \& Ainsworth, B. E. (2016). Recruiting participants into pilot trials: Techniques for researchers with shoestring budgets. Californian Journal of Health Promotion, 14(2), 81-89.

Koerting, J., Smith, E., Knowles, M. M., Latter, S., Elsey, H., McCann, D. C. et al. (2013). Barriers to, and facilitators of, parenting programmes for childhood behaviour problems: A qualitative synthesis of studies of parents' and professionals'perceptions. European Child and Adolescent Psychiatry, 22(11), 653-670. https://doi.org/10.1007/ s00787-013-0401-2

Lim, M., Stormshak, E. A., \& Dishion, T. J. (2005). A one-session intervention for parents of young adolescents: Videotape modeling and motivational group discussion. Journal of Emotional and Behavioral Disorders, 13(4), 194-199. https://doi.org/10.1177/10634266050130040101

Löfgren, H. O., Petersen, S., Nilsson, K., Ghazinour, M., \& Hägglöf, B. (2017). Effects of parent training programmes on parents' sense of competence in a general population sample. Global Journal of Health Science, 9(7), 24-34. https://doi.org/10.5539/gjhs.v9n7p24

Marinho, M. L. (2005). Um programa estruturado para o treinamento de pais. In V. E. Caballo, \& M. A. Simon (Eds.), Manual de psicologia clínica infantil e do adolescente (pp. 417-433). São Paulo, SP: Santos.

McCurdy, K., \& Daro, D. (2001). Parent involvement in family support programs: An integrated theory. Family Relations, 50(2), 113-121. https://doi.org/10.1111/j.1741-3729.2001.00113.x

Mencarelli, V. L., \& Aiello-Vaisberg, T. M. J. (2012). Cuidado emocional na saúde pública: A psicologia clínica ampliada. In D. F. Gioia-Martins (Ed.), Psicologia e saúde, formação, pesquisa e prática profissional (pp.45-64). São Paulo, SP: Vetor.

Miyazaki, M., Domingos, N., Valerio, N., Santos, A., \& Rosa, L. (2002). Psicologia da saúde: Extensão de serviços à comunidade, ensino e pesquisa. Psicologia USP, 13(1), 29-53. https://doi.org/10.1590/S0103-65642002000100003

Mytton, J., Ingram, J., Manns, S., \& Thomas, J. (2014). Facilitators and barriers to engagement in parenting programs: A qualitative systematic review. Health Education \& Behavior, 41(2), 127-137. https://doi. org/10.1177/1090198113485755

Oliveira, J. M., \& Alvarenga, P. (2015). Efeitos de uma intervenção com foco nas práticas de socialização parentais sobre os problemas internalizantes na infância. Revista Brasileira de Terapia Comportamental e Cognitiva, 17(2), 16-32. https://doi.org/10.31505/rbtcc.v17i2.747

Paulon, S. M. (2005). A análise de implicação como ferramenta na pesquisa-intervenção. Psicologia \& Sociedade, 17(3), 18-25. https://doi.org/10.1590/S0102-71822005000300003

Pozzobon, M., Falcke, D., \& Marin, A. (2018). Intervenção com famílias de alunos com baixo desempenho escolar. Ciências Psicológicas, 12(1), 87-96. https://doi.org/10.22235/cp.v12i1.1599

Pupo, D. (2015). Projeto de intervenção para aumentar a adesão ao tratamento de hipertensão no PSF estreito município Água Branca (Trabalho de conclusão). Universidade Federal de Minas Gerais, Maceió, AL, Brasil.

Rolfsen, A. B., \& Martinez, C. M. S. (2008). Programa de intervenção para pais de crianças com dificuldades de aprendizagem: Um estudo preliminar. Paidéia (Ribeirão Preto), 18(39), 175-188. https://doi.org/10.1590/S0103863X2008000100016

Santos, M. A. M. I. B., Nasser, A. C. A., Basso, C. R., \& Paiva, V. S. (2013). Intervenção em adesão baseada na abordagem construcionista do cuidado: Perspectiva dos profissionais de saúde. Temas em Psicologia, 21(3), 651-673. https://doi.org/10.9788/TP2013.3-EE02PT

Smith, E., Koerting, J., Latter, S., Knowles, M. M., McCann, D. C., Thompson, M. et al. (2015). Overcoming barriers to effective early parenting interventions for attention-deficit hyperactivity disorder (ADHD): Parent and practitioner views. Child: Care, Health and Development, 41(1), 93-102. https://doi.org/10.1111/cch.12146

Stormshak, E. A., Connell, A. M., Véronneau, M., Myers, M.W., Dishion, T. J., Kavanagh, K. et al. (2011). An ecological approach to promoting early adolescent mental health and social adaptation: Family-centered intervention in public middle schools. Child Development, 82(1), 209-225. https://doi.org/10.1111/j.1467-8624.2010.01551.x

Straub, R. O. (2014). O papel da psicologia da saúde nos cenários de atendimento à saúde. In R. O. Straub, Psicologia da saúde: Uma abordagem biopsicossocial (3a ed., pp. 340-369). Porto Alegre, RS: Artes Médicas.

Szymanski, H. \& Cury, V. E. (2004). A pesquisa intervenção em psicologia da educação e clínica: Pesquisa e prática psicológica. Estudos de Psicologia (Natal), 9(2), 355-364. https://doi.org/10.1590/S1413-294X2004000200018 
Tramm, R., Daws, K., \& Schadewaldt, V. (2013). Clinical trial recruitment: A complex intervention? Journal of Clinical Nursing, 22(17/18), 2436-2443. https://doi.org/10.1111/jocn.12145

Weeland, J., Chhangur, R. R., Giessen, D., Matthys, W., Castro, B. O., \& Overbeek, G. (2017). Intervention effectiveness of the icredible years: New insights into sociodemographic and intervention-based moderators. Behavior Therapy, 48(1), 1-18. https://doi.org/10.1016/j.beth.2016.08.002

\section{Angela Helena Marin}

Doutora em Psicologia. professora e pesquisadora nos cursos de Graduação e Pós-graduação em Psicologia da Universidade Federal do Rio Grande do Sul (UFRGS), Porto Aelgre - RS. Brasil.

E-mail: marin.angelah@gmail.com

(D) http://orcid.org/0000-0002-8056-8661

\section{Patrícia Alvarenga}

Doutora em Psicologia e professora do Programa de Pós-graduação em Psicologia da Universidade Federal da Bahia (UFBA), Salvador - BA. Brasil.

E-mail:palvarenga66@gmail.com

(iD) http://orcid.org/0000-0001-9079-4956

\section{Magda Pozzobon}

Mestre em Psicologia pelo Programa de Pós-graduação em Psicologia da Universidade do Vale do Rio dos Sinos (UNISINOS), São Leopoldo - RS, Brasil.

E-mail: magdapozzobon@hotmail.com

$$
\text { http://orcid.org/0000-0002-8938-173X }
$$

\section{Taiane Costa de Souza Lins}

Mestre e Doutoranda em Psicologia pelo Programa de Pós-graduação em Psicologia da Universidade Federal da Bahia (UFBA), Salvador - BA. Brasil.

E-mail: lins.taiane@gmail.com

(iD) http://orcid.org/0000-0001-6411-8620

\section{João Marcos de Oliveira}

Mestre e Doutorando em Psicologia pelo Programa de Pós-graduação em Psicologia da Universidade Federal da Bahia (UFBA), Salvador - BA. Brasil.

E-mail: joaomarcosdeoliveira@gmail.com

(iD) http://orcid.org/0000-0001-5494-3985

Endereço para envio de correspondência:

Rua Ramiro Barcelos, 2600 - sala 221. Bairro Santa Cecília.

CEP: 90035-003. Porto Alegre - RS Brasil.

Recebido 30/10/2017

Reformulado 10/07/2018

Aceito 10/09/2018

Received 10/30/2017

Reformulated $07 / 10 / 2018$

Approved 09/10/2018 
Psicologia: Ciência e Profissão 2019 v. 39, e187233, 1-14.

Recibido 30/10/2017

Reformulado 10/07/2018

Aceptado 10/09/2018

Como citar: Marin, A. H., Alvarenga, P., Pozzobon, M., Lins, T. C. S., \& Oliveira, J. M. (2019). Evasão em intervenções psicológicas com pais de crianças e adolescentes: relato de experiência. Psicologia: Ciência e Profissão, 39, 1-14. https:// doi.org/10.1590/1982-3703003187233

How to cite: Como citar: Marin, A. H., Alvarenga, P., Pozzobon, M., Lins, T. C. S., \& Oliveira, J. M. (2019). Dropout in psychological interventions with parents of children and adolescents: experience report. Psicologia: Ciência e Profissão, 39, 1-14. https://doi.org/10.1590/1982-3703003187233

Cómo citar: Marin, A. H., Alvarenga, P., Pozzobon, M., Lins, T. C. S., \& Oliveira, J. M. (2019). Evasión en intervenciones psicológicas con padres de niños y adolescentes: informe de experiencia. Psicologia: Ciência e Profissão, 39, 1-14. https:// doi.org/10.1590/1982-3703003187233 